\title{
Visual direction constancy across eyeblinks
}

\author{
J. Stephen Higgins, David E. Irwin, \\ RanXiao Frances Wang, and Laura E. Thomas \\ University of Illinois at Urbana-Champaign, Urbana, Illinois
}

\begin{abstract}
When a visual target is displaced during a saccade, the perception of its displacement is suppressed. Its movement can usually only be detected if the displacement is quite large. This suppression can be eliminated by introducing a short blank period after the saccade and before the target reappears in a new location. This has been termed the blanking effect and has been attributed to the use of otherwise ignored extraretinal information. We examined whether similar effects occur with eyeblinks and other visual distractions. We found that suppression of displacement perception can also occur due to a blink (both immediately prior to the blink and during the blink), and that introducing a blank period after a blink reduces the displacement suppression in much the same way as after a saccade. The blanking effect does not occur when other visual distractions are used. This provides further support for the conclusion that the blanking effect arises from extraretinal signals about eye position.
\end{abstract}

Blinks and saccadic eye movements occupy more of our day-to-day life than perhaps any other human behavior, but because of the visual suppression generated by these behaviors we very rarely realize we are making these movements at all. This is a good thing, because without visual suppression we would live in a world of constant visual blurs and voids. Early explorations into saccadic suppression discovered that people are able to encode text only during brief periods of fixation while reading and are unable to gather meaning from the text for the duration of an eye movement (Erdmann \& Dodge, 1898). Another often cited example of saccadic suppression is our inability to see our own eye movements in a mirror (Dodge, 1900; Erdmann \& Dodge, 1898; see Bridgeman, Hendry, \& Stark, 1975, for a quantitative resolution of this issue).

Eye movements present a serious challenge the brain needs to overcome in order to perceive a stable visual world and interact with it. When an object appears in one place on our retina, then appears in another after an interruption, how does the visual system recognize that it is the same object, in the same place as before, and now just located on a new retinal position? Three pieces of information must be present in order to maintain visual constancy and to successfully determine the direction of a target displacement if one has occurred: memory for the position of the target prior to the saccade, information about the direction and amplitude of the eye movement, and knowledge about the position of the target after the saccade. Together these three pieces of information allow a viewer to determine whether a visual target has moved and in which direction.

Information about the direction and amplitude of an eye movement may come from any of three independent sources (Bridgeman, Van der Heijden, \& Velichkovsky,
1994). The first is the structure of the external visual environment where objects, surfaces, or other visual cues (motion, etc.) can be used as reference points (Gibson, 1950, 1966, 1979; Haber, 1983). This is typically called retinal information, because it consists of visual information on the retina. The second is motor planning and the commands that are issued to the ocular motor systemthat is, neural outflow, efference copy, or corollary discharge (Sperry, 1950). The last type of spatial information is the proprioceptive information from receptors in the eye movement muscles, that is, neural inflow (e.g., Sherrington, 1918). The latter two sources of information are typically called extraretinal because they rely on nonvisual sources of information, namely, ocular motor information.

Using these three (or sometimes fewer) sources of information, a number of theories have been proposed to solve the challenge of perceiving a stable visual world. These include an elimination solution (Sperry, 1950; Von Holst \& Mittelstaedt, 1950/1980, 1971), a translation solution (Bischof \& Kramer, 1968), an evaluation solution (MacKay, 1973), a calibration solution (Bridgeman et al., 1994), and a localist evaluation solution (Currie, McConkie, Carlson-Radvansky, \& Irwin, 2000; Irwin, McConkie, Carlson-Radvansky, \& Currie, 1994; cf. Deubel \& Schneider, 1994; Deubel, Schneider, \& Bridgeman, 1996). Each solution has its own strengths and weaknesses (see Bridgeman et al., 1994, and its accompanying commentaries for a good summary of theories of visual direction constancy).

From the studies evaluating each of these theories it appears that the degree to which neural inflow, neural outflow, and visual scene information contribute to the perception of a stable visual world depends on the cir-

J. S. Higgins, higgins3@illinois.edu 
cumstances of the environment. Visual scene information, when present, appears to dominate inflow and outflow cues in determining whether visual stability is achieved (Deubel, 2004; Deubel et al., 1996; Matin et al., 1982). When no visual scene information is present, however, the only useful source of information is extraretinal. This leads to some surprising consequences.

Previous research has shown that people are quite poor at detecting whether the position of a visual target presented in isolation is displaced around the time of a saccade; target displacements as large as $10 \%-25 \%$ of saccade amplitude go frequently undetected (Bridgeman et al., 1975). This phenomenon, called saccadic suppression of image displacement (SSID), is characterized by the inability to detect changes in the location of a target when the change occurs immediately before, during, or shortly after the saccade, and follows a time course very similar to that of the suppression of visual sensitivity that accompanies saccades (e.g., Volkmann, 1986). The magnitude of SSID (approximately $4 \log$ units) is much larger than the reduction in visual sensitivity (0.5-0.7 log units) that accompanies saccades (Bridgeman et al., 1975; Volkmann, 1986).

These results indicate that, when it comes to spatially localizing visual targets across saccades, our perceptual system neglects (or is unable to use) extraretinal information about eye position. Surprisingly, in contrast to the perceptual system, it appears that the motor system is able to access precise spatial information in order to render precise motor actions (Bridgeman, Lewis, Heit, \& Nagle, 1979; Prablanc \& Martin, 1992). If precise spatial information is available to our motor system, is there any way for the perceptual system to gain access to it in order to make more accurate displacement judgments?

The answer appears to be yes; under certain conditions, people gain access to motor information in order to make more accurate perceptual judgments. For example, if a target displaced during a saccade is not visible at the end of the saccade but reappears a short time later after a blank interval, subjects are able to regain the ability to successfully detect whether the target has moved (Deubel, Bridgeman, \& Schneider, 1998; Deubel et al., 1996). In these studies, it was discovered that, as the time between the end of the saccade and the presentation of the shifted target increased, participants became much more accurate at detecting displacement, and that, with a delay of $200 \mathrm{msec}$, participants reached ceiling in their performance. Deubel and colleagues termed this the blanking effect, and its existence suggests that it is used only when visual scene information is not available, although extraretinal information is present and contains accurate location information. In a follow-up study, a blink replaced the blank at the end of a saccade; under these circumstances no blanking effect was found, demonstrating that voluntary blinks do not produce the same effect as blanking of the stimulus after a saccade (Deubel, Bridgeman, \& Schneider, 2004). Thus, when it comes to visual direction constancy, a blink is not a blank, but might potentially be more akin to a saccade.

Although visual direction constancy across saccadic eye movements has been studied extensively for over a century, to our knowledge no one has investigated visual direction constancy across eyeblinks. Even though we blink, on average, every $4 \mathrm{sec}$, or 900 times in an hour (Ponder \& Kennedy, 1927), we very rarely notice that we have blinked at all. In terms of visual input, blinking is equivalent to turning out all of the lights in a room for a short time. Despite this, we do not experience a complete blackout of our vision during a blink, much in the same way that we do not perceive a blur across our visual field during a saccade (Riggs, Volkmann, \& Moore, 1981). Thus, researchers have wondered whether there are common neural mechanisms underlying blinks and saccades. Indeed, blink-induced suppression of visual thresholds has been equated very well to saccadic suppression in both time course and magnitude of suppression (Volkmann, 1986; Volkmann, Riggs, Ellicott, \& Moore, 1982; Volkmann, Riggs, \& Moore, 1980). A number of neuroimaging studies also suggest that blink suppression and saccadic suppression are associated with activity in the same brain areas (Bristow, Frith, \& Rees, 2005; Bristow, Haynes, Sylvester, Frith, \& Rees, 2005; Kleiser, Seitz, \& Krekelberg, 2004).

Despite the similarities between blinks and saccades, it is not obvious that the same mechanisms that contribute to visual direction constancy across saccades would also operate across eyeblinks. Saccades cause the fovea to move from one position in space to another, necessitating some mechanism to compensate for or evaluate this movement; in contrast, the position of the fovea after a blink is more or less identical to its position before, so no compensatory or evaluative mechanism may be necessary to maintain perceptual constancy; in fact, it is even possible that visual direction constancy is not even maintained across a blink. Recent research has shown that blinking interferes with iconic memory by disrupting the binding between object position and object identity (Thomas \& Irwin, 2006), so perhaps memory for spatial information is simply lost across blinks. Furthermore, changing the contents of a visual display during a blink often goes unnoticed (O'Regan, Deubel, Clark, \& Rensink, 2000), providing further evidence that memory across blinks may be poor. Thus, it is simply not known whether visual direction constancy operates across blinks and, if it does, whether it has the same characteristics as visual direction constancy across saccades.

To examine this issue, we observed whether target displacements are detected across blinks and whether a blanking effect occurs across blinks as it does across saccades. To explore further the processes involved, we also examined whether other visual disruptions (in addition to those associated with saccades and blinks) might interfere with spatial localization. In sum, in the present experiments we investigated the following three specific questions:

1. Is suppression of target displacement perception specific to saccades, or does it occur during blinks as well?

2. If suppression of target displacement perception occurs in response to a blink, can the suppression be eliminated in the same way as it can be eliminated after a saccade - by inserting a delay at the end of a blink before the target is re-presented? 
3. Is suppression of target displacement perception specific to saccades or blinks, or will any visual disruption cause suppression of target displacement perception?

\section{EXPERIMENT 1}

In this experiment, we sought to determine whether blinking reduces people's accuracy in detecting target displacements and also whether this suppression is similar to that of saccadic suppression in that it can be elicited by a blink-related efference signal. On each trial a single dot was presented on the display for $100 \mathrm{msec}$, then extinguished; then, either 50 or $750 \mathrm{msec}$ later, the dot was re-presented above, below, left of, or right of its initial position. The subjects' task was to indicate the direction of displacement. Performance was compared across two experimental conditions. In one, participants were instructed to blink as soon as they saw the first dot; in the other, they kept their eyes open. If perception of target displacement is suppressed by blinking, accuracy should be lower in the blink condition than in the no-blink condition. If this occurs even before the eyes start to blink (i.e., on trials where an ISI of only $50 \mathrm{msec}$ separates the two presentations of the target dot), this would indicate that merely the intention to blink (i.e., blink motor programming) is sufficient to suppress the perception of target displacement, consistent with neural outflow hypotheses of visual direction constancy.

\section{Method}

Participants. Eight students from the University of Illinois were recruited for this experiment. Participants reported normal or corrected-to-normal vision and were naive as to the purpose of the experiment. They received payment for participation. None of the students participated in the other experiments.

Apparatus. Stimuli were presented on a 21 -in. monitor with resolution of $800 \times 600$ pixels and a refresh rate of $85 \mathrm{~Hz}$. Eye movements and blinks were recorded with an Eyelink II videobased eyetracker (SR Research Ltd., Mississauga, Ontario, Canada) with temporal resolution of $500 \mathrm{~Hz}$, spatial resolution of $0.1^{\circ}$, and pupil-size resolution of $0.1 \%$ of pupil diameter. The output of the eyetracker was parsed online by Eyelink II analysis software. Each data sample from the eyetracker contained a timestamp in milliseconds, the velocity and the position of the eye, and the area of the pupil. The parser computed instantaneous velocity and acceleration for each sample and compared these to velocity and acceleration thresholds for saccade detection. A change in eye position was classified as a saccade when its distance exceeded $0.2^{\circ}$ and its velocity reached $30^{\circ} / \mathrm{sec}$, or when its distance exceeded $0.2^{\circ}$ and its acceleration reached $9,500^{\circ} / \mathrm{sec}$. The eyetracker software defined an eyeblink as a period of missing pupil for at least 6 consecutive msec that was preceded and followed by a period of artifactual saccade caused by the motion of the eyelids across the pupil. Blink onset and blink offset were defined to correspond to the beginning and ending of this artifactual saccade. Fixations were defined as any period that was not a blink or a saccade. Custom C code was written to display stimuli and collect responses. Participants' heads were stabilized with a chinrest, fixed at $48 \mathrm{~cm}$ from the computer monitor. Responses were gathered with a gamepad (Microsoft, Redmond, WA). The room was well lit, so the edges of the computer monitor and other visual landmarks were visible. Each experimental block of trials began with a nineposition calibration procedure, in which the edges and center of the screen were fixated; a drift-correction procedure was completed at the beginning of each experimental trial.
Procedure. Figure 1 shows the sequence of events on a typical trial. Participants were asked to focus on the drift-correct dot at the beginning of each trial and to press a button on the response pad to begin the trial. After the drift-correct dot disappeared, a blank white screen was presented for $500 \mathrm{msec}$, after which a red target dot was presented for $100 \mathrm{msec}$. The six possible initial target locations were arranged in a $2.9^{\circ}$ circle centered on the original location of the driftcorrect dot. On blink trials, participants were instructed to blink as soon as they saw the target appear on the screen, whereas on noblink trials they were instructed to keep their eyes open throughout the trial. The target dot was re-presented either 50 or $750 \mathrm{msec}$ after the end of its initial presentation; the re-presented target was presented for $100 \mathrm{msec}$, displaced either above, below, left of, or right of its original position by $0.71^{\circ}$. Participants were asked to report in which direction the target had moved.

On blink trials, the 50-msec interstimulus interval (ISI) between target presentations constituted a preblink condition; that is, the target dot was re-presented before the participant initiated a blink. All trials in which the participant started to blink before or during the presentation of the displaced target were excluded from analysis. The blink trials that had a 750-msec ISI between target presentations constituted a postblink condition; that is, the blink was executed between target presentations (trials in which this did not occur were deleted from analysis). The no-blink trials with 50- and 750-msec ISIs provided control conditions against which any deleterious effects of blink programming and/or blink execution could be measured; noblink trials in which blinks or saccades occurred were deleted from analysis. Each participant completed three blocks of blink trials (48 trials per block) and three blocks of no-blink trials. Order was counterbalanced across participants. The ISI-50 and ISI-750 conditions were sequenced randomly within each block. The first block of both the no-blink trials and the blink trials was considered to be practice and was not analyzed.

\section{Results and Discussion}

Preliminary analyses showed that there were no systematic differences in accuracy due to initial target position or direction of displacement, so to increase power, the data were averaged over these variables. Mean blink latency in the ISI-50 condition was $309 \mathrm{msec}(S D=20 \mathrm{msec})$, whereas it was $303 \mathrm{msec}(S D=18 \mathrm{msec})$ in the ISI-750 condition. Mean blink duration in the ISI-50 condition was $236 \mathrm{msec}(S D=46 \mathrm{msec})$, and was $214 \mathrm{msec}(S D=$ $38 \mathrm{msec}$ ) in the ISI-750 condition.

The accuracy results are shown in Figure 2. Not surprisingly, accuracy was higher when a short delay $(50 \mathrm{msec})$ separated target presentations $(M=96.6 \%)$ than when a long delay $(750 \mathrm{msec})$ separated them $(M=71.1 \%)$ $\left[F(1,7)=93.4, M S_{\mathrm{e}}=55.4, p<.001\right]$. More importantly, accuracy was lower on blink $(M=80.4 \%)$ than on noblink trials $(M=87.4 \%)\left[F(1,7)=21.9, M S_{\mathrm{e}}=17.9\right.$, $p<.002]$. The interaction between blink condition and delay was not significant $\left[F(1,7)=1.3, M S_{\mathrm{e}}=59.2, p>\right.$ $.25]$, demonstrating that blinking significantly affected accuracy at both short and long delays.

These data demonstrate that perception of visual displacement is suppressed by blinks in much the same fashion as by saccades, both immediately prior to a blink as well as after the completion of a blink. The ISI-750 condition demonstrates that blinks interrupt the internal representation of target location above and beyond the normal degradation of target location information caused by the passage of time. It is noteworthy that in the ISI-50 condition the target dot was presented and re-presented 


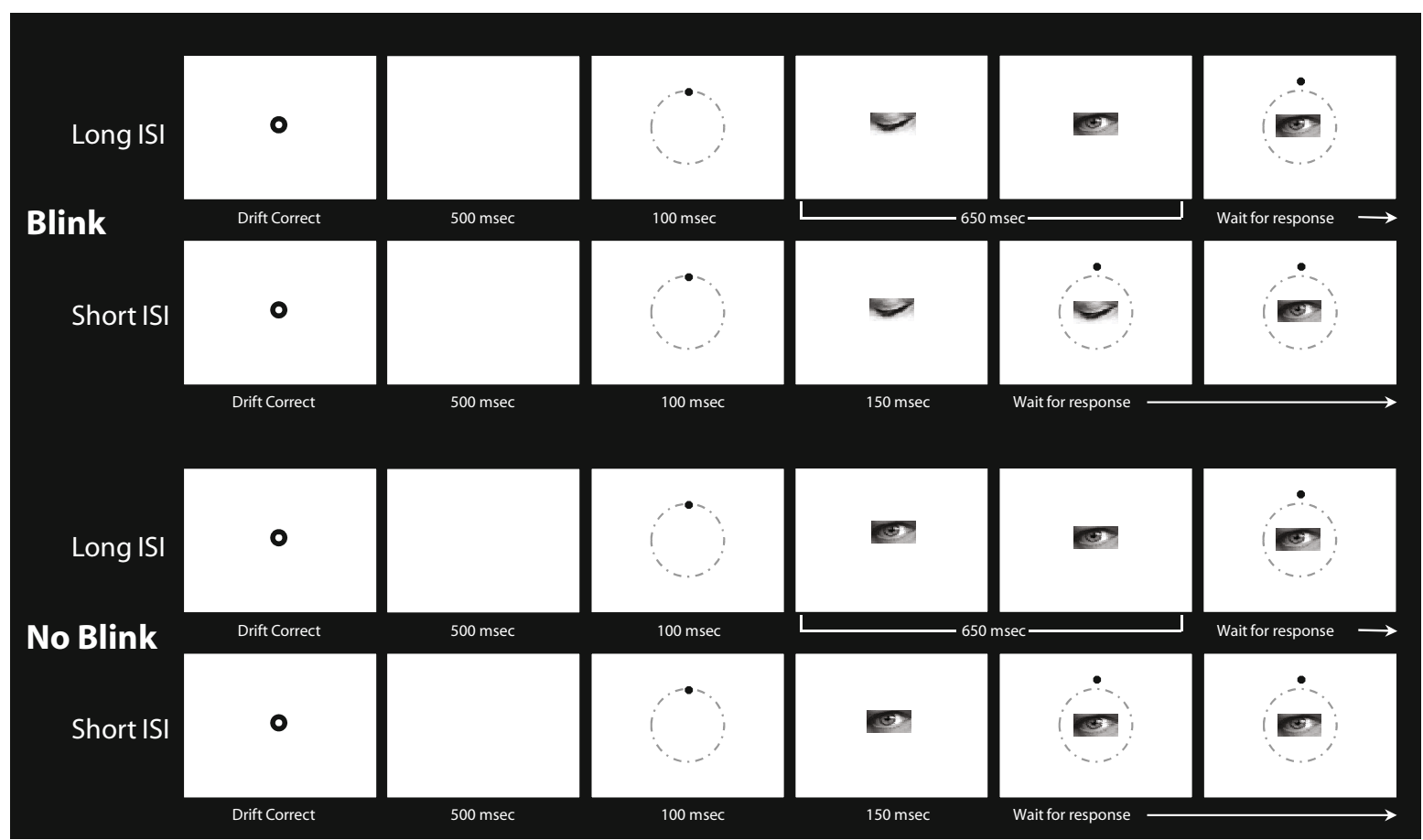

Figure 1. Experiment 1 trials. Trials began with a drift-correction fixation display. The participants were instructed to remain fixated during the entire trial. In blink trials, participants were instructed to blink as soon as the target appeared on the screen. In no-blink trials, participants were instructed to keep their eyes where they originally fixated. In most cases the target disappeared either immediately before participants blinked, or while their eyes were closed. The target could be displaced up, down, left, or right during the ISI/blink. As soon as participants opened their eyes, they were instructed to report in which direction the target had moved.

before the blink started, but errors in displacement detection still occurred. The fact that suppression was found even before the blink started indicates that blink planning and programming (neural outflow) is sufficient to cause suppression of target displacement perception. Retinal information (scene landmarks) and perhaps proprioceptive (neural inflow) information also contributed to task performance, however, as demonstrated by the fact that overall accuracy was well above chance even though blink planning and execution interfered with memory for target dot location. As we described at the beginning of this article, the same factors influence saccadic suppression of image displacement.

It is well known that the eyes themselves move during an eyeblink (e.g., Bour, Aramideh, \& Ongerboer de Visser, 2000; Collewijn, van der Steen, \& Steinman, 1985; Evinger, Shaw, Peck, Manning, \& Baker, 1984). For example, Collewijn et al. reported that during voluntary and reflexive eyeblinks, both eyes consistently move down and toward the nose, with amplitudes in the range of $1^{\circ}$ to $5^{\circ}$. After an analysis of their properties (e.g., duration, velocity), Collewijn et al. concluded that these blink-related eye movements are not saccades; rather, they seem to occur as a result of the eyeball being retracted during the eyeblink (see also, Bour et al., 2000; Evinger et al., 1984). Given that blink-related eye movements are not saccades, it seems unlikely that the suppression of the perception of visual displacement that we observed on blink trials in this experiment was due to saccadic suppression rather than to blink suppression.

Although the small eye movements that occur during a blink are not saccades (Collewijn et al., 1985), it is still of interest to determine whether these movements may have influenced performance in our task. Because the eyes move during a blink, their point of fixation after the blink is often not identical to what it was before the blink. If the blink suppression that we observed in this experiment was actually due to changes in eye position across the blink, one would expect the accuracy of displacement detection to be affected by the direction and/or the magnitude of the change in fixation position before and after the blink. For example, participants should make more "right" responses when their fixation position moves toward the left across the blink than when it moves toward the right (direction effect), and they should have lower accuracy when the change in fixation position across the blink is larger rather than smaller (magnitude effect).

To examine the direction effect, we compared the fixation position of the eyes after the blink to what it had been before, and coded any change in fixation position as being predominantly left, right, up, or down. Then we compared the probability of a given response (e.g., "right") when the fixation position changed across the blink in the same direction as the target displacement (right) to when it changed in the opposite direction as the target displacement (left) or in an orthogonal direction (up or down, in 




Figure 2. Mean accuracy in Experiment 1. Error bars represent standard errors of the means.

this case). If changes in fixation position were to influence the accuracy of detecting a target displacement it would be expected that fixation changes in the same direction as the target displacement would cause a decrement in accuracy, because the change in fixation position would essentially cancel out some of the actual target displacement on the retina. Similarly, fixation position changes in the opposite or orthogonal directions from the target displacement would be expected to increase detection of displacement, because the effective target displacement on the retina would be larger. To test this, an ANOVA was conducted on the accuracy of displacement detection with direction of fixation position change (same as, opposite from, or orthogonal to target displacement direction) as the sole factor. The direction of fixation position change had no effect on accuracy $[F(2,21)=0.167]$. When the fixation position changed across the blink in the same direction as the target displacement, accuracy was $75 \%$; when it changed in the opposite direction as target displacement, accuracy was $72 \%$; when it changed in an orthogonal direction with respect to target displacement, accuracy was $74 \%$. These results indicate that changes in fixation position (and, hence, eye movement) across a blink have no effect on the detection of target displacement.

To examine the magnitude effect, we coded changes in fixation position across the blink into three categories according to their size: Those that moved less than the target displacement distance $\left(0.71^{\circ}\right)$, those that moved between $0.71^{\circ}$ and $1.42^{\circ}$, and those that moved more than $1.42^{\circ}$. The mean change in fixation position for these three categories was $0.45^{\circ}, 1.07^{\circ}$, and $2.31^{\circ}$, respectively. Mean displacement detection accuracy for the trials that fell into these three categories was $79 \%, 75 \%$, and $71 \%$. Although this pattern is in the expected direction, a one-way ANOVA examining the effect of the size of the fixation position change on displacement detection accuracy did not approach significance $[F(2,21)=0.93]$.

Overall, these analyses suggest that there is no relationship between the direction or magnitude of blink-related eye movements and the accuracy of perceived target displacement. This indicates that the suppression of the perception of target displacement is due to the blink itself and not to any accompanying changes in eye position. In sum, the answer to the first question that we posed at the beginning of this article is that suppression of target displacement perception occurs for blinks as well as for saccades.

\section{EXPERIMENT 2}

The purpose of Experiment 2 was to determine whether suppression of displacement perception can be eliminated after a blink in the same way as it can be eliminated after a saccade - by inserting a delay at the end of a blink before the target is re-presented. Recall that Deubel et al. (1996) called this the blanking effect. To demonstrate whether a blanking effect can occur after a blink, the timing of stimulus presentations was manipulated in Experiment 2 to create a condition in which target displacement occurred during the blink (so that when the eyes reopened, the second target dot was already visible) and another condition in which the target was presented well after the blink was over (so that a temporal delay separated blink termination and presentation of the second dot). If the blanking effect occurs for blinks as well as for saccades, participants should be more accurate at detecting displacements in the condition where the target reappears well after the blink is over.

\section{Method}

Participants. Sixteen people from the introductory psychology participant pool took part in this study for course credit. Participants reported normal or corrected-to-normal vision and were naive as to the purpose of the experiment. None of the students participated in the other experiments.

Procedure. Figure 3 shows the sequence of events on a typical trial. Participants were asked to focus on the drift-correct dot at the beginning of each trial and to press a button on the response pad to begin the trial. After the drift-correct dot disappeared, a blank white 


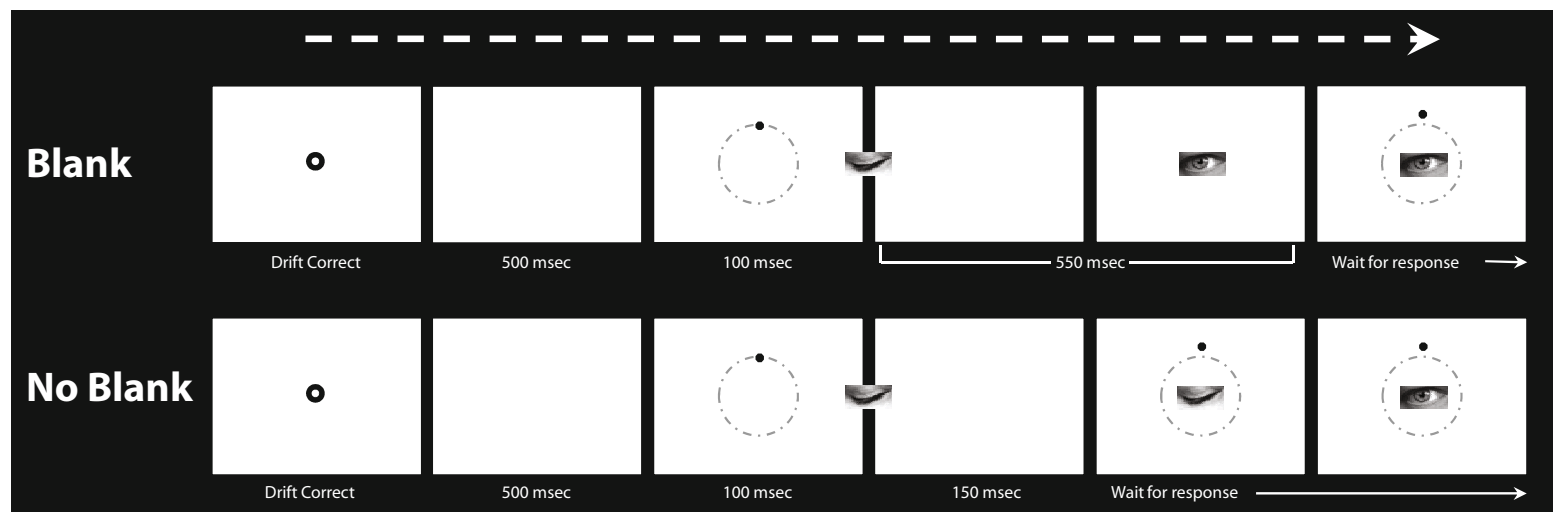

Figure 3. Experiment 2 trials. Trials began with a drift-correction fixation display. Participants were instructed to blink as soon as the target appeared. In most cases, the target disappeared while the participants' eyes were closed. The target could be displaced up, down, left, or right during the blink. When participants opened their eyes, either the target was present (no blank) or there was nothing on the screen until a short time later when the target reappeared (blank). As soon as the target reappeared, participants were instructed to report in which direction it had moved.

screen was presented for $500 \mathrm{msec}$, after which a red target dot was presented for $100 \mathrm{msec}$. The six possible initial target locations were arranged on an invisible $2.9^{\circ}$ circle centered on the original location of the drift-correct dot. Participants were instructed to blink as soon as they saw the target appear on the screen.

In the ISI-250 condition, the target dot was re-presented $250 \mathrm{msec}$ after the end of its initial presentation, usually while the participants' eyes were closed. The target was displaced either above, below, left of, or right of its original position by $0.71^{\circ}$. In the ISI- 650 condition, the displaced dot was displayed $650 \mathrm{msec}$ after the disappearance of the original target. At this point on most trials, participants had their eyes open for $\sim 350 \mathrm{msec}$ before seeing the onset of the displaced target. The displaced target remained visible until the participant indicated which direction the target had moved in. Each participant completed six blocks of 48 trials each. The ISI-250 and ISI- 650 conditions were sequenced randomly within each block. The first block of trials was considered to be practice and was not analyzed.

\section{Results and Discussion}

Average blink latency was $240 \mathrm{msec}(S D=181 \mathrm{msec})$. Average blink duration was $226 \mathrm{msec}(S D=101 \mathrm{msec})$. Thus, as intended, on most of the ISI-250 trials the target dot was re-presented during the blink, while the eyes were closed, so that when the eyes reopened the target dot was visible on the screen (i.e., there was no blank before the target reappeared). Also as intended, on most of the ISI-650 trials the target dot was re-presented well after $(\sim 350 \mathrm{msec})$ the eyes reopened from the blink (i.e., there was a blank before the target reappeared). Because the stimulus presentation timings were fixed, however, a number of different trial types resulted from participants' variable blinking behavior. The two most common were the ones described above: ISI-250 trials in which no blank occurred, and ISI-650 trials in which a blank occurred. There were also ISI-250 trials in which a blank occurred (because subjects blinked so quickly that their blink ended before the presentation of the displaced target) and, infrequently, ISI-650 trials in which no blank occurred (because of a long-latency blink). There were also many trials in which no blink occurred or a saccade was executed; these trials were not analyzed. The first three trial types, which we will call ISI-250 no-blank, ISI-650 blank, and
ISI-250 blank, were the only ones analyzed, because ISI650 no-blank trials occurred rarely and were not present for many subjects. Participants with fewer than 30 occurrences of any trial type of interest were eliminated from further analysis. In the end, 11 of the 16 participants were used for analysis.

The results from Experiment 2 are presented in Figure 4. The results demonstrate a reduction of displacement suppression in the two blank conditions. This is comparable to the saccade experiments of Deubel et al. (1996). Participants were less accurate on ISI-250 no-blank trials $(54.3 \%)$ than on ISI-650 blank trials $(65.4 \%)$ or on ISI-250 blank trials (63.0\%). The two types of blank trials were not significantly different from each other $[t(10)<1$, n.s.], whereas both the ISI-650 blank trials and the ISI-250 blank trials were significantly greater than the ISI-250 noblank trials $[t(10)=3.802, p<.003$, and $t(10)=4.707$, $p<.001$, respectively].

In the experiments of Deubel et al. (1996), it was shown that suppression decreased (i.e., accuracy increased) as the duration of the blank increased from 0 to $200 \mathrm{msec}$ (at which point accuracy reached asymptote). To further compare the blink-related elimination of displacement suppression to that of saccades, an analysis was undertaken to determine whether increased blank duration between the end of the blink and the presentation of the displaced target positively affected displacement detection. Because participant-generated blank durations were roughly comparable to those examined by Deubel et al. (1996), we median-split trials for each participant on the basis of the blank duration of that participant, in order to determine whether there was a positive relationship between blank duration and accuracy. There was, in fact, a significant relationship between blank duration and accuracy, with accuracy increasing from $60 \%$ correct when participants' blank duration was below the median $(M=62 \mathrm{msec})$ to $69 \%$ when above $[t(10)=2.813, p<.018]$.

This experiment presents further information about the suppression of displacement perception during blinks. In particular, it demonstrates that this suppression can be re- 


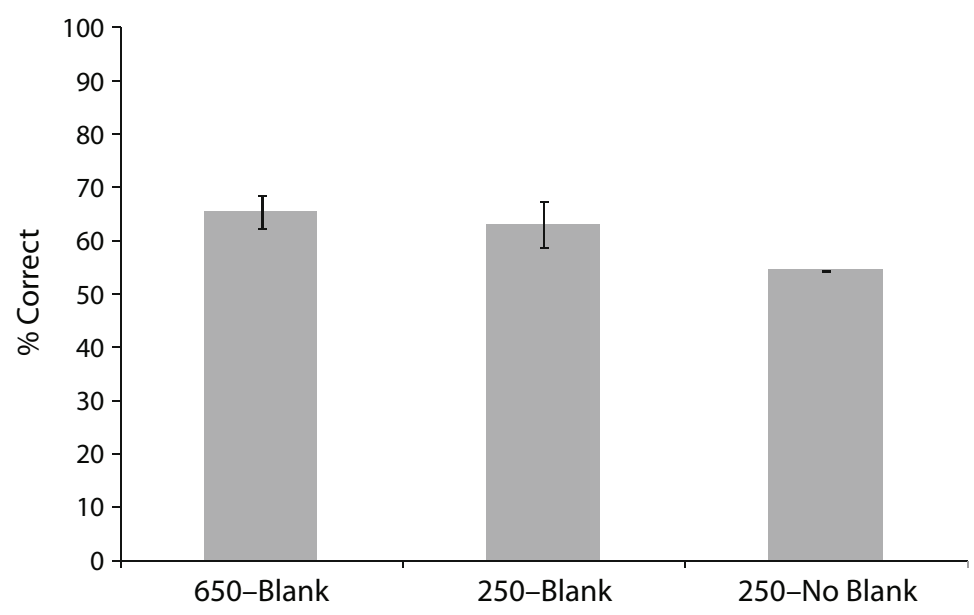

Figure 4. Mean accuracy in Experiment 2. Error bars represent standard errors of the means.

duced in the same way in which saccadic suppression of image displacement can be reduced - by inserting a blank before the target reappears after blink or saccade offset. This suggests that extraretinal information may be used for visual direction constancy across eyeblinks as well as across saccades; that is, as Deubel et al. (1996) have proposed, when the visual target is present after a saccade or an eyeblink (as on no-blank trials), the perceptual system assumes that its position has not changed and does not access, or ignores, extraretinal information (i.e., neural outflow or neural inflow) about eye position. When a blank is present, however, the perceptual system has nothing else to work with, so it makes use of extraretinal information about eye position to calculate the spatial position of the target, thereby allowing perception of target displacement.

An alternative explanation for the blanking effect observed in this experiment needs to be considered, however. Blinks are visually disruptive events that may interfere with the processing of other visual stimuli presented in close temporal proximity (e.g., Thomas \& Irwin, 2006). On no-blank trials, the target stimulus was present immediately after the blink, whereas on blank trials the target did not reappear until approximately $300 \mathrm{msec}$ after the blink. The longer delay on blank trials may have allowed the perceptual system to recover from the visually disruptive effects of the blink and thereby facilitate performance. In other words, the benefit in performance for blank over no-blank trials may have been due to the recovery of function with the passage of time after any generic distraction, rather than to the use of extraretinal information. This possibility was examined in Experiment 3.

\section{EXPERIMENT 3}

In this experiment, we examined whether a simulated blink would also interfere with spatial memory, and whether performance would improve when a blank period followed the simulated blink. If the blanking effect observed in Experiment 2 was due to the recovery of function with the passage of time following a blink, accuracy should be higher in this experiment when a blank follows a simulated blink than when it does not.

\section{Method}

Participants. Ten people from the introductory psychology participant pool took part in this study for course credit. Participants had normal or corrected-to-normal vision and were naive as to the purpose of the experiment. None of them participated in the other experiments.

Procedure. The experimental procedure for simulated blink trials was identical to that of Experiment 2, except that, instead of blinking, participants were presented with a black screen that simulated the perceptual consequences of a blink. On each trial a red target dot was presented for $100 \mathrm{msec}$, at one of six possible initial target locations arranged in a $2.9^{\circ}$ circle in the center of the display. The target dot was re-presented either 250 or $650 \mathrm{msec}$ later, having been moved either up, down, left, or right of its original position by $0.71^{\circ}$. On simulated blink trials the screen changed from white to black $150 \mathrm{msec}$ after the termination of the initial target dot (corresponding to a blink latency of $250 \mathrm{msec}$, similar to that found in Experiment 2), and remained black for a total of $150 \mathrm{msec}$, corresponding closely to the amount of time that the pupil was covered by the eyelids in Experiment 2. After the simulated blink, the screen turned from black to white and remained that way until the subject responded. On ISI-250 trials, the re-presented target dot was visible as soon as the simulated blink ended, producing a no-blank trial. On ISI-650 trials, the target dot was re-presented $350 \mathrm{msec}$ after the simulated blink ended, producing a blank trial. Two (no simulated blink) control conditions were included that had the same timing as the simulated blink conditions, but with a white screen displayed instead of a black screen (see Figure 5). Participants were asked to report the direction in which the target moved from its original position, as in the first two experiments.

\section{Results and Discussion}

The results are presented in Figure 6. A $2 \times 2$ repeated measures ANOVA with distraction (simulated blink vs. no simulated blink) and ISI as fixed factors revealed that the simulated blink interfered with performance (mean accuracy of $67 \%$ vs. $73 \%)\left[F(1,9)=19.96, M S_{\mathrm{e}}=.003, p=\right.$ $.002]$. The main effect of ISI was not significant, however (identical mean accuracy $70 \%)\left[F(1,9)=0.77, M S_{\mathrm{e}}=\right.$ $.002]$, and neither was the interaction between ISI and distraction $\left[F(1,9)=1.850, M S_{\mathrm{e}}=.003\right]$. 


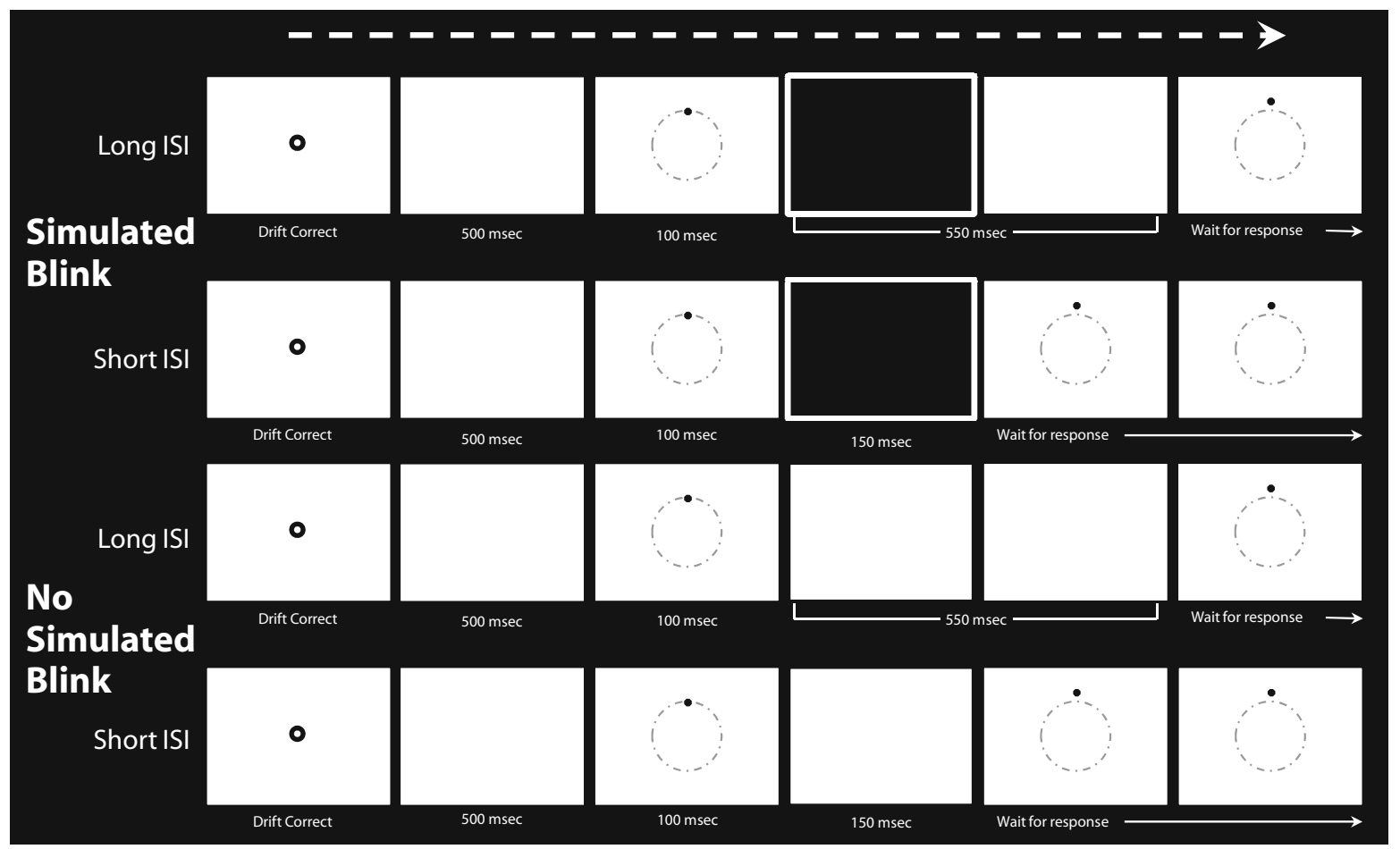

Figure 5. Experiment 3 trials. Trials began with a fixation display. Participants were instructed to fixate during the entire trial. During the simulated blink trials, the screen would go black in the ISI; during the other trials a blank white screen was present. The target could be displaced up, down, left, or right during the blink. As soon as the target reappeared, participants were instructed to report in which direction it had moved.

We conducted several other versions of Experiment 3 using several other kinds of visual disruptions (e.g., moving grids instead of a black field), or physical disruptions (e.g., pressing a button instead of blinking) and found that accuracy for target displacement detection decreased as the disruption became more distracting, but no blanking effect was ever discovered.

These results are inconsistent with the hypothesis that the blanking effect found in Experiment 2 was due to the recovery of function with the passage of time following an eye blink. Even though the simulated eyeblink reduced accuracy in Experiment 3 almost to the same extent as real eyeblinks reduced accuracy in Experiment 1 (6\% vs. $7 \%$ ), there was no benefit in performance for blank as opposed to no-blank trials in Experiment 3. Thus, the results of Experiment 2 seem unlikely to be due to the visual consequences of blinking (e.g., the disappearance of the visual field), but rather are more likely to be due to extraretinal sources of eye position information being used to calculate spatial position during the blank, facilitating displacement perception.

\section{GENERAL DISCUSSION}

The purpose of the present research was to investigate whether visual direction constancy operates across eyeblinks, and, if so, to determine whether it has some of the same characteristics as visual direction constancy across saccades. In Experiment 1, we examined whether people could discriminate the direction that a visual target was displaced during an eye blink and whether the blink and its programming interfered with discrimination performance. In Experiment 2, we examined whether the blanking effect occurs across blinks as it does across saccades. In Experiment 3, we examined whether visual disruptions that mimic those of blinks have effects on performance similar to those of blinks per se.

Experiment 1 demonstrated that visual direction constancy does operate across eyeblinks: People were able to judge with high accuracy in which direction a visual target object moved across an eyeblink. Because this experiment was conducted in a well-lit room, any one or more of three possible sources of information might have contributed to this: visual landmarks, neural outflow, or neural inflow. Accuracy was higher when people did not blink than when they did, however, indicating that suppression of target displacement perception occurs for blinks as well as for saccades. The fact that suppression was found even before the blink started (i.e., in the 50-msec ISI condition) indicates that neural outflow (i.e., blink planning and programming) was one source of information. Similar results have been found for saccades (e.g., Matin, 1986). However, the decrement to displacement detection was not as great as when observers actually completed a blink, indicating at the very least that each stage or aspect of blink programming and execution influences the suppression of displacement detection.

Experiment 2 showed that suppression of displacement perception can be eliminated after a blink in the same way 


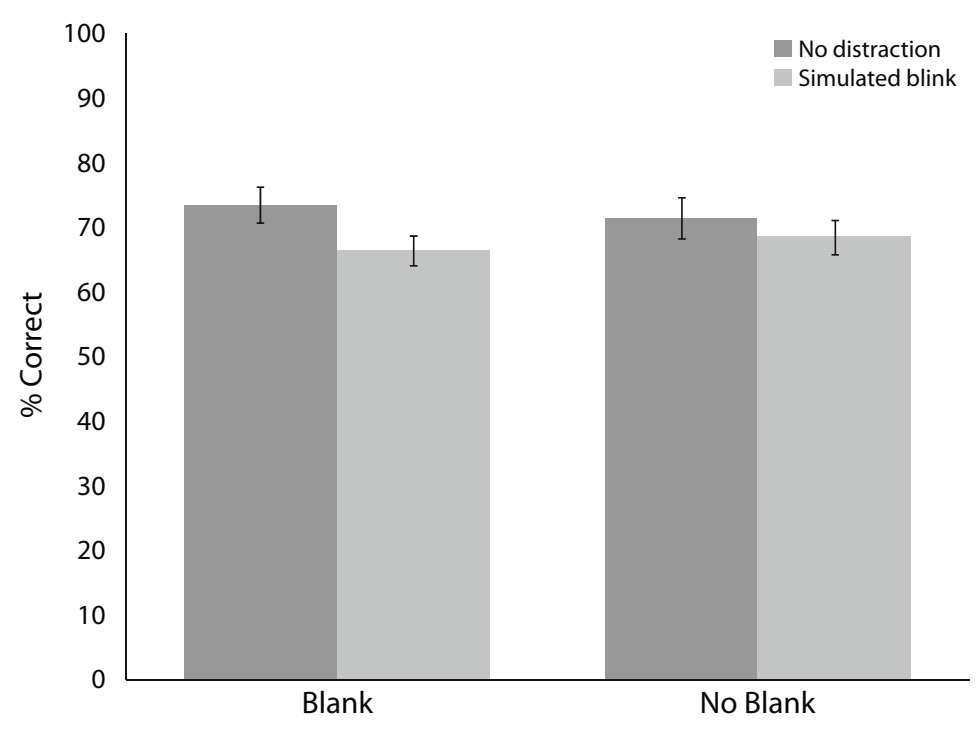

Figure 6. Mean accuracy in Experiment 3. Error bars represent standard errors of the means.

as it can be eliminated after a saccade-by inserting a blank period at the end of the blink before the target is re-presented. That is, participants were more accurate at detecting target displacements when the target reappeared well after the blink was over than when the target was visible immediately after blink offset. Similar results have been found for saccades, as well as for blinks preceded by saccades (Deubel et al., 2004). Why should a blank period improve, rather than impair, performance? Deubel and colleagues have proposed that extraretinal information about eye position (neural outflow and/or neural inflow) is available after eye movements and contains accurate localization information, but it is not used when visual scene information is present. That is, although accurate extraretinal information is available, in the presence of visual scene information the perceptual system is biased to assume that target position has not changed and so it does not access, or simply ignores, the extraretinal information. This is consistent with many other studies that have shown that visual scene information dominates inflow and outflow cues in determining visual direction constancy (Deubel, 2004; Deubel et al., 1996; Matin et al., 1982). However, when a blank appears and no visual scene information is present, the assumption of target stability is broken, and the perceptual system makes use of the extraretinal information to calculate the spatial position of targets, allowing much more accurate displacement detection. The results of Experiment 2 provide support for the conclusion that visual direction constancy across eyeblinks depends on a similar mechanism.

Experiment 3 demonstrated that a simulated blink interfered with target displacement perception, but did not produce a blanking effect. These results are consistent with the hypothesis that the effects of blinking are due not just to the visual consequences of a blink, but also to extraretinal sources of eye-position information. By comparing results from Experiments 2 and 3, we may be able to gain further insight into what sources of information contribute to the suppression. When observers made no eye movements and encountered no distractions, but simply stared at the stimulus as it disappeared and reappeared in a new location, they were on average $74.8 \%$ correct. However, when they encountered a distraction simulating a blink, their accuracy dropped to a mean of $65.8 \%$ correct (Experiment 3, blank conditions). When observers made a blink with no blank afterward, they were only $54.3 \%$ correct (Experiment 2, no blank-250 condition), but if a blank was added after the blink, they were $65.4 \%$ correct (Experiment 2, blank-650 condition). By adding a blank period after a blink, observers were just as accurate as if they only had encountered a brief distraction that simulated a blink (a black screen) $[t(19)=0.14$, n.s.]. Participants in the blanking condition were still significantly less accurate in an unpaired $t$ test than if they had not encountered a visual distraction at all $[t(19)=2.3, p<.03]$. This result suggests that adding a blank can eliminate displacement suppression caused by blink planning and execution (nonretinal information) but not from the visual distraction that it causes (retinal information).

Our results demonstrate that the perception of stability across eyeblinks relies on the same kind of information that supports perceptual stability across saccades, namely, visual landmarks that provide information about the relative positions of objects in the environment as well as extraretinal information about eye position based on neural inflow and neural outflow. When visual landmarks are present, they appear to dominate inflow and outflow cues. In the absence of visual landmarks, however, the extraretinal information is accessed to help determine target position. The initial (premovement) position of the target must also be retained in memory for purposes of comparison with the final (postmovement) position of the target so that displacement can be detected (Hollingworth, Richard, \& Luck, 2008). 
As with most investigations, a number of questions remain to be answered. For example, it would be good to know the relative contributions of inflow, outflow, and retinal scene information to visual direction constancy across blinks. At a minimum, experiments would need to be conducted in total darkness to eliminate the presence of all visual scene information. Manipulations such as paralyzing the oculomotor muscles to eliminate inflow but not outflow information would also be necessary (see Matin, 1986, and Bridgeman et al., 1994, for discussions of analogous experiments conducted with saccades).

Another question requiring further investigation is whether suppression of target displacement perception and recovery during a postblink blanking interval occurs during reflexive eyeblinks as well as during the voluntary eyeblinks that we investigated. Reflexive eyeblinks occur involuntarily, in response to some external stimulus (such as a puff of air directed at the cornea), whereas voluntary eyeblinks are made in response to endogenous factors or experimental instruction. It is known that visual sensitivity is suppressed before, during, and after an eyeblink (Volkmann, 1986; Volkmann et al., 1982; Volkmann et al., 1980) and that the magnitude and time course of this suppression is nearly identical for reflexive and voluntary eyeblinks (Manning, Riggs, \& Komenda, 1983). On the basis of this finding, Manning et al. concluded that blink-induced visual suppression is due to a low-level efferent pathway common to both reflexive and voluntary eyeblinks. The same might well be true for blink-induced suppression of displacement perception. Voluntary blinks require cortical involvement, so finding a difference between reflexive and voluntary blinks in our task would indicate that suppression of displacement perception involves cortical processing, whereas suppression of visual sensitivity does not. We plan to investigate this in future research.

Finally, it would be interesting to compare the magnitude of blink suppression with that of saccadic suppression, but such a comparison would be difficult to carry out. One problem involves equating stimulus quality across blink and saccade conditions. In our experiments the pre- and postblink stimuli both appeared in the parafovea and were separated by only $0.71^{\circ}$ on the display screen; in order to study the saccadic suppression of image displacement, however, the pre- and postsaccadic stimuli are typically presented several degrees apart in space and occupy quite different retinal locations (periphery and fovea). Another problem involves equating blink amplitude and saccade amplitude; the threshold for detecting target displacements across saccades depends on saccade amplitude (e.g., Bridgeman et al., 1975), and it may well be the case that the same will be true for blink amplitudes. Because the eye movements that accompany blinks are not saccades (Bour et al., 2000; Collewijn et al., 1985; Evinger et al., 1984); however, it is not obvious how to relate blink amplitudes to saccade amplitudes. Testing a variety of target displacements across a range of blink and saccade amplitudes and comparing the resulting psychometric functions will be required. This is another possible avenue for future research.
Although many questions remain to be answered, the results of the present research demonstrate that suppression of displacement perception occurs for blinks (both immediately prior to the blink and during the blink) as has previously been demonstrated for saccades, that introducing a blank period after a blink reduces the displacement suppression in much the same way as has been demonstrated for saccades, and that this blanking effect does not occur when other visual distractions are used, providing further support for the conclusion that the blanking effect arises from extraretinal signals about eye position. Our results indicate that visual direction constancy across eyeblinks relies on the same kind of information that supports visual direction constancy across saccades.

\section{AUTHOR NOTE}

This research was supported by NSF Grant BCS 03-17681 to R.F.W. The authors thank Jeremy Wolfe, Heiner Deubel, Bruce Bridgeman, and one anonymous reviewer for their suggestions on an earlier version of the article. We also thank the many undergraduate students who helped in running this experiment. Correspondence concerning this article should be addressed to J. S. Higgins, Department of Psychology, University of Illinois, 603 E. Daniel St., Champaign, IL 61820 (e-mail: higgins3@ illinois.edu)

\section{REFERENCES}

BischoF, N., \& KRAMER, E. (1968). Untersuchungen und überlegungen zur Richtungswahrnehmung bei willkürlichen sakkadischen Augenbewegungen. Psychologische Forschung, 32, 185-218.

Bour, L. J., Aramideh, M., \& Ongerboer de Visser, B. W. (2000). Neurophysiological aspects of eye and eyelid movements during blinking in humans. Journal of Neurophysiology, 83, 166-176.

Bridgeman, B., Hendry, D., \& Stark, L. (1975). Failure to detect displacement of the visual world during saccadic eye movements. Vision Research, 15, 719-722.

Bridgeman, B., Lewis, S., Heit, G., \& Nagle, M. (1979). Relation between cognitive and motor-oriented systems of visual position perception. Journal of Experimental Psychology: Human Perception \& Performance, 5, 692-700.

Bridgeman, B., Van der Heijden, A. H. C., \& Velichkovsky, B. M. (1994). A theory of visual stability across saccadic eye movements. Behavioral \& Brain Sciences, 17, 247-292.

Bristow, D., Frith, C., \& Rees, G. (2005). Two distinct neural effects of blinking on human visual processing. Neurolmage, 27, 136-145.

Bristow, D., Haynes, J. D., Sylvester, R., Frith, C. D., \& Rees, G. (2005). Blinking suppresses the neural response to unchanging retinal stimulation. Current Biology, 15, 1296-1300.

Collewijn, H., van der Steen, J., \& Steinman, R. M. (1985). Human eye movements associated with blinks and prolonged eyelid closure. Journal of Neurophysiology, 54, 11-27.

Currie, C., McConkie, G. W., Carlson-Radvansky, L. A., \& Irwin, D. E. (2000). The role of the saccade target object in the perception of a visually stable world. Perception \& Psychophysics, 62, 673-683.

Deubel, H. (2004). Localization of targets across saccades: Role of landmark objects. Visual Cognition, 11, 173-202.

Deubel, H., Bridgeman, B., \& Schneider, W. X. (1998). Immediate postsaccadic information mediates space constancy. Vision Research, 38, 3147-3159.

Deubel, H., Bridgeman, B., \& Schneider, W. X. (2004). Different effects of eyelid blinks and target blanking on saccadic suppression of displacement. Perception \& Psychophysics, 66, 772-778.

Deubel, H., \& Schneider, W. X. (1994). Can man bridge a gap? Behavioral \& Brain Sciences, 17, 259-260.

Deubel, H., Schneider, W. X., \& Bridgeman, B. (1996). Postsaccadic target blanking prevents saccadic suppression of image displacement. Vision Research, 36, 985-996.

Dodge, R. (1900). Visual perception during eye movement. Psychological Review, 7, 454-465. 
Erdmann, B., \& Dodge, R. (1898). Psychologische untersuchungen uber das lesen auf experimenteller grundlage. Halle, Germany: Niemeyer.

Evinger, C., Shaw, M. D., Peck, C. K., Manning, K. A., \& BaKer, R. (1984). Blinking and associated eye movements in human, guinea pigs and rabbits. Journal of Neurophysiology, 52, 323-339.

GiBson, J. J. (1950). The perception of the visual world. Boston: Houghton Mifflin.

GiBson, J. J. (1966). The senses considered as perceptual systems. Boston: Houghton Mifflin.

GiBson, J. J. (1979). The ecological approach to visual perception. Boston: Houghton Mifflin

Haber, R. N. (1983). The impending demise of the icon: A critique of the concept of iconic storage in visual information processing. Behavioral \& Brain Sciences, 6, 1-54.

Hollingworth, A., Richard, A. M., \& LUCK, S. J. (2008). Understanding the function of visual short-term memory: Transsaccadic memory, object correspondence, and gaze correction. Journal of Experimental Psychology: General, 137, 163-181.

Irwin, D. E., McConkie, G. W., Carlson-Radvansky, L. A., \& CurRIE, C. (1994). A localist evaluation solution for visual stability across saccades. Behavioral \& Brain Sciences, 17, 265-266.

Kleiser, R., Seitz, R. J., \& Krekelberg, B. (2004). Neural correlates of saccadic suppression in humans. Current Biology, 14, 386-390.

MACKAY, D. M. (1973). Visual stability and voluntary eye movements. In R. Jung (Ed.), Handbook of sensory physiology (Vol. 7, pp. 307332). New York: Springer.

Manning, K. A., Riggs, L. A., \& Komenda, J. K. (1983). Reflex eyeblinks and visual suppression. Perception \& Psychophysics, 34, 250-256.

Matin, L. (1986). Visual localization and eye movements. In K. R. Boff, L. Kaufman, \& J. P. Thomas (Eds.), Handbook of perception and human performance (Vol. 1, pp. 20-1 to 20-45). New York: Wiley.

Matin, L., Picoult, E., Stevens, J. K., Edwards, M. W., JR., Young, E., \& MacArThur, R. (1982). Oculoparalytic illusion: Visual-field dependent spatial mislocations by humans partially paralyzed with curare. Science, 216, 198-201.
O’Regan, K., Deubel, H., Clark, J. J., \& Rensink, R. A. (2000). Picture changes during blinks: Looking without seeing and seeing without looking. Visual Cognition, 7, 191-211.

Ponder, E., \& Kennedy, W. P. (1927). On the act of blinking. Quarterly Journal of Experimental Physiology, 18, 89-110.

Prablanc, C., \& Martin, O. (1992). Automatic control during hand reaching at undetected two-dimensional target displacements. Journal of Neurophysiology, 67, 455-469.

Riggs, L. A., Volkmann, F. C., \& Moore, R. K. (1981). Suppression of the blackout due to blinks. Vision Research, 21, 1075-1079.

Sherrington, C. S. (1918). Observations on the sensual role of the proprioceptive nerve supply of the extrinsic eye muscles. Brain, $\mathbf{4 1}$, 332-343.

SPERRY, R.W. (1950). Neural basis of the spontaneous optokinetic response produced by visual inversion. Journal of Comparative \& Physiological Psychology, 43, 482-489.

Thomas, L. E., \& IRwin, D. E. (2006). Voluntary eyeblinks disrupt iconic memory. Perception \& Psychophysics, 68, 475-488.

Volkmann, F. C. (1986). Human visual suppression. Vision Research, 26, 1401-1416.

Volkmann, F. C., Riggs, L. A., Ellicott, A. G., \& Moore, R. K. (1982). Measurements of visual suppression during opening, closing and blinking of the eyes. Vision Research, 22, 991-996.

Volkmann, F. C., Riggs, L. A., \& Moore, R. K. (1980). Eyeblinks and visual suppression. Science, 207, 900-902.

Von Holst, E., \& Mittelstaedt, H. (1971). The principle of reafference: Interactions between the central nervous system and the peripheral organs. In P. C. Dodwell (Ed.), Perceptual processing: Stimulus equivalence and pattern recognition (pp. 41-47). New York: Appleton.

Von Holst, E., \& Mittelstaedt, H. (1980). The reafference principle. In C. R. Gallistel (Ed.), The organization of action (pp. 176-207). New York: Wiley. (Original work published 1950)

(Manuscript received July 23, 2008; revision accepted for publication May 23, 2009.) 